\title{
Enantioselective Amination of $\alpha$-Phenyl- $\alpha$-cyanoacetate Catalyzed by Chiral Amines Incorporating the a-Phenylethyl Auxiliary
}

Yongjun Liu, Roberto Melgar-Fernández, and Eusebio Juaristi ${ }^{*}$

Departamento de Química, Centro de Investigación y de Estudios Avanzados del Instituto Politécnico

Nacional, Apartado postal 14-740, 07000 México, D.F., Mexico.

e-mail: juaristi@relaq.mx

\section{Supporting Information}

\section{Contents}

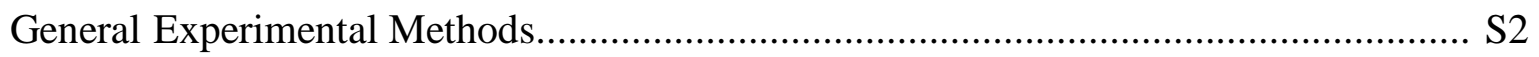

General procedure for enantioselective amination.................................................. S2

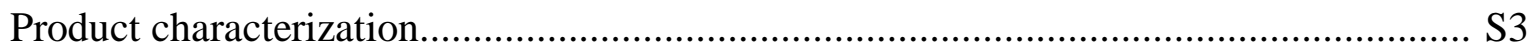

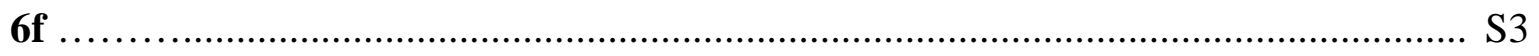

6e

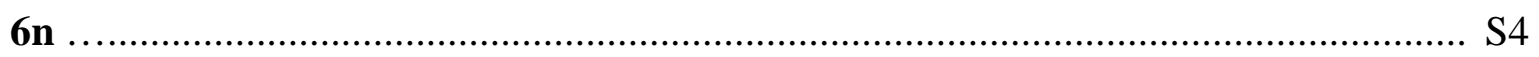

6p

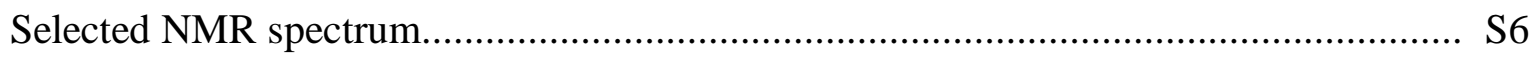

Selected HPLC chromatograms.............................................................................. S13 
General Methods. Melting points: not corrected. ${ }^{1} \mathrm{H}$ NMR spectra: $400 \mathrm{MHz}$ spectrometer. ${ }^{13} \mathrm{C}$ NMR: $100 \mathrm{MHz}$ spectrometer. Chemical shifts $(\delta)$ in $\mathrm{ppm}$ downfield from internal TMS reference; the coupling constants $(J)$ are given in Hz. Optical rotations were measured in a polarimeter, using the sodium D-line $(589 \mathrm{~nm})$. Mass spectra: $20 \mathrm{eV}$. All reactions were carried out under a nitrogen atmosphere. Toluene was distilled from sodium and hexane was also distilled before use. Crude products were purified by flash chromatography on silica gel 230-400 meshes. High performance liquid chromatography (HPLC) analysis using a Chiralpak AD Column (250 x $4.6 \mathrm{~mm})$, hexane-isopropanol 85:15 as eluents, $220 \mathrm{~nm} \mathrm{UV} \mathrm{detector,} 1.0 \mathrm{~mL} / \mathrm{min}$. Ethyl $\alpha$-phenyl- $\alpha$-cyanoacetate (1), di-tert-butyl azodicarboxylate (2), (DHQ) ${ }_{2} \mathrm{PYR} \quad(\mathbf{4 a})$, $(\mathrm{DHQD})_{2} \mathrm{PYR}(\mathbf{4 b}),(-)$-sparteine (5), and $(R)$ - $\alpha$-methylphenylethylamine (6a) are commercially available and were used as received. Compounds $6 \mathbf{b},{ }^{1} \mathbf{6 c},{ }^{1} 6 \mathbf{6},{ }^{1} \mathbf{6 g},{ }^{2} 6 \mathbf{6},{ }^{2 \mathrm{~b}} 6 \mathbf{6},{ }^{3 \mathrm{a}} 6 \mathbf{6},{ }^{3 \mathrm{a}} \mathbf{6 k},{ }^{3 \mathrm{~b}} 6 \mathbf{6},{ }^{4} 6 \mathbf{6 m},{ }^{4}$ $\mathbf{6 0},{ }^{5}$ were synthesized by literature procedures.

General procedure for enantioselective amination, 3. A mixture of ethyl $\alpha$-phenyl- $\alpha$ cyanoacetate $(0.55 \mathrm{mmol})$ and catalyst $(0.25 \mathrm{mmol})$ in $2 \mathrm{~mL}$ of toluene (or $9 \mathrm{~mL}$ toluene) was stirred for $1 \mathrm{~h}$ at room temperature, and then was cooled to $-78{ }^{\circ} \mathrm{C}$ (or $-100{ }^{\circ} \mathrm{C}$ ) under a nitrogen atmosphere. A solution of di-tert-butyl azodicarboxylate $(0.5 \mathrm{mmol}$ ) in $1 \mathrm{~mL}$ of toluene (or $2 \mathrm{~mL}$ of toluene-hexane, 1:1 v/v) was added dropwise over a period of $30 \mathrm{~min}$. The reaction mixture was stirred until the yellow color of the solution faded (10 min - $30 \mathrm{~h}$ ), and then allowed to warm to room temperature. Pure products were isolated by flash chromatography (ethyl acetate / hexane, 1:6). ${ }^{1} \mathrm{H}$ NMR (400 MHz, DMSO) $\delta 1.20(\mathrm{t}, J=6.9 \mathrm{~Hz}, 3 \mathrm{H}), 1.33(\mathrm{~s}, 9 \mathrm{H}), 1.48(\mathrm{~s}, 9 \mathrm{H})$, $4.24(\mathrm{q}, J=6.9,2 \mathrm{H}), 7.45(\mathrm{~s}, 3 \mathrm{H}), 7.63(\mathrm{~s}, 2 \mathrm{H}), 8.70(\mathrm{br}, \mathrm{N}-\mathrm{H}) .{ }^{13} \mathrm{C}$ NMR $(100 \mathrm{MHz}, \mathrm{DMSO}) \delta$ $13.9,28.3,28.5,63.7,80.8,80.9,83.6,116.3,127.2,128.0,128.6,129.1,130.5,130.7,153.9$, 154.9, 165.4, see attached spectrum. Enantiomeric excess determined by HPLC $\left[t_{\mathrm{R}}(-)\right.$ levo 
enantiomer $=11 \mathrm{~min} ; t_{\mathrm{R}}(+)$ dextro enantiomer $\left.=37 \mathrm{~min}\right) . \mathrm{mp}: 94-96{ }^{\circ} \mathrm{C} . \mathrm{IR}\left(\mathrm{CH}_{2} \mathrm{Cl}_{2}\right) \vee\left(\mathrm{cm}^{-1}\right)$ : $3337,2982,2360,1747,1716,1370,1243,1154,1053,[\alpha]_{\mathrm{D}}^{20}=+48.7\left(c=1, \mathrm{CHCl}_{3}\right)$ for $84 \%$ ee. Literature: ${ }^{6}[\alpha]_{\mathrm{D}}^{20}=+64.0\left(c=0.175, \mathrm{CHCl}_{3}\right)$ for $97 \%$ ee.

General procedure for $6 \mathbf{e}$ and $6 \mathbf{6 f}$. To a solution of $2.82 \mathrm{~g}(10 \mathrm{mmol})$ of diamine $6 \mathbf{c}$ or $6 \mathbf{d}$ in 10 $\mathrm{mL}$ of toluene, was added $0.36 \mathrm{~g}(12 \mathrm{mmol})$ of paraformaldehyde at room temperature. The mixture was slowly heated to reach reflux temperature within $1 \mathrm{~h}$ and then heated to reflux for 4 h. The solvent was evaporated over vacuum and the product was isolated by flash chromatography (ethyl acetate/hexane, 1:3) before to distillation $\left(160^{\circ} \mathrm{C} / 0.5 \mathrm{mmHg}\right)$.

(S,S)-1,3-bis-(1-phenyl-ethyl)-hexahydro-pyrimidine (6e). Yield 77\% as a light yellow oil. $[\alpha]_{\mathrm{D}}^{20}=-82.9\left(c=1.3, \mathrm{CHCl}_{3}\right) ;{ }^{1} \mathrm{H} \mathrm{NMR}\left(400 \mathrm{MHz}, \mathrm{CDCl}_{3}\right) \delta 1.31(\mathrm{~d}, J=6.6 \mathrm{~Hz}, 6 \mathrm{H}), 1.63(\mathrm{dt}$, $J=5.5,10.6 \mathrm{~Hz}, 2 \mathrm{H}), 2.51(\mathrm{br}, 4 \mathrm{H}), 3.24(\mathrm{~s}, 2 \mathrm{H}), 3.56(\mathrm{q}, J=6.6 \mathrm{~Hz}, 2 \mathrm{H}), 7.21-7.30(\mathrm{~m}, 10 \mathrm{H})$; ${ }^{13} \mathrm{C}$ NMR $\left(100.5 \mathrm{MHz}, \mathrm{CDCl}_{3}\right) \delta 20.0,23.3,49.9,61.6,71.3,126.9,127.7,128.2,144.4 . \mathrm{IR}$ (neat) $v\left(\mathrm{~cm}^{-1}\right): 3026,2971,2801,1947,1877,1807,1754,1674,1452,1280,1111,761,700$. HRMS: (TOF-ESI) m/z calcd for $\mathrm{C}_{20} \mathrm{H}_{26} \mathrm{~N}_{2} \mathrm{H}(\mathrm{M}+\mathrm{H})^{+} 295.2168$, found 295.2169.

$(\boldsymbol{R}, \boldsymbol{R})-\mathbf{1 , 3}-$ Bis-(1-phenyl-ethyl)-hexahydro-pyrimidine (6f). Yield $84 \%$ as a light yellow oil. $[\alpha]_{\mathrm{D}}^{20}=+87.6\left(c=2, \mathrm{CHCl}_{3}\right) ;{ }^{1} \mathrm{H} \mathrm{NMR}\left(270 \mathrm{MHz}, \mathrm{CDCl}_{3}\right) \delta 1.28(\mathrm{~d}, J=6.6 \mathrm{~Hz}, 6 \mathrm{H}), 1.60$ $(\mathrm{dt}, J=5.5,10.9 \mathrm{~Hz}, 2 \mathrm{H}), 2.49(\mathrm{dd}, J=4.9,10.4 \mathrm{~Hz}, 4 \mathrm{H}), 3.22(\mathrm{~s}, 2 \mathrm{H}), 3.54(\mathrm{q}, J=6.5 \mathrm{~Hz}, 2 \mathrm{H})$, 7.19 - $7.32(\mathrm{~m}, 10 \mathrm{H}) ;{ }^{13} \mathrm{C} \mathrm{NMR}\left(67.9 \mathrm{MHz}, \mathrm{CDCl}_{3}\right) \delta 19.9,23.1,49.8,61.5,71.2,126.8,127.6$, 128.1, 144.1. IR (neat) $v\left(\mathrm{~cm}^{-1}\right): 3026,2972,2801,1946,1876,1808,1753,1675,1452,1280$, 1111, 761, 700. MS (EI) m/z (\%) $295\left(12, \mathrm{M}^{+}+1\right), 294\left(53, \mathrm{M}^{+}\right), 293\left(100, \mathrm{M}^{+}-1\right), 189(24)$, 105 (61), 85 (64); Anal. Calcd. For $\mathrm{C}_{20} \mathrm{H}_{26} \mathrm{~N}_{2}$ (294.43): C, 81.59; H, 8.90; N, 9.51. Found: C, 81.72; H, 9.29; N, 9.63. 
(S)-Benzyl-(1-naphthalen-1-yl-ethyl)-amine (6n). According to the procedure described in the literature, ${ }^{5}$ a solution of $1.71 \mathrm{~g}(10 \mathrm{mmol})$ of $(S)$-1-naphthalen-1-yl-ethylamine in DMPU (20 mL) was treated with $2.12 \mathrm{~g}$ of $\mathrm{Na}_{2} \mathrm{CO}_{3}(20 \mathrm{mmol})$, and $2.05 \mathrm{~g}(12 \mathrm{mmol})$ of benzyl bromide was added dropwise. The mixture was stirred at $100{ }^{\circ} \mathrm{C}$ for $4 \mathrm{~h}$ and then cooled down in an ice bath before the addition of water $(50 \mathrm{~mL})$. The reaction product was extracted with ethyl acetate $(3 \mathrm{x}$ $50 \mathrm{~mL}$ ), the combined organic phase was washed with water $\left(3 \times 20 \mathrm{~mL}\right.$ ), dried on $\mathrm{Na}_{2} \mathrm{SO}_{4}$, filtered and concentrated under vacuum. The product was isolated by flash chromatography (ethyl acetate/hexane, 1:6), and distilled $\left(180{ }^{\circ} \mathrm{C}, 0.5 \mathrm{mmHg}\right)$ to give $2.28 \mathrm{~g}(83 \%$ yield $)$ of $\mathbf{6 n}$ as colorless oil. Optical rotation of hydrochloric acid salt: $[\alpha]_{\mathrm{D}}^{20}=+61.7^{\circ}(c=1.6, \mathrm{MeOH})\left[\right.$ Lit. $^{7 \mathrm{a}}$ $[\alpha]_{\mathrm{D}}^{20}=-61.0(c=2, \mathrm{MeOH})$ for $R$ enantiomer]; ${ }^{1} \mathrm{H} \mathrm{NMR}\left(400 \mathrm{MHz}, \mathrm{CDCl}_{3}\right)\left[\right.$ Lit. $\left.^{7 \mathrm{~b}, \mathrm{c}}\right] \delta 1.54(\mathrm{~d}$, $J=6.6 \mathrm{~Hz}, 3 \mathrm{H}), 1.76(\mathrm{br}, \mathrm{N}-\mathrm{H}), 3.74(\mathrm{~d}, J=13.2 \mathrm{~Hz}, 1 \mathrm{H}), 3.81(\mathrm{~d}, J=13.2 \mathrm{~Hz}, 1 \mathrm{H}), 4.72(\mathrm{q}, J=$ 6.6 Hz, 1H), 7.25-7.36 (m, 5H), 7.50-7.55 (m, 3H), 7.78-7.80 (m, 2H), 7.89-7.92 (m, 1H), 8.18 $(\mathrm{m}, 1 \mathrm{H}) .{ }^{13} \mathrm{C} \mathrm{NMR}\left(75 \mathrm{MHz}, \mathrm{CDCl}_{3}\right) \delta 24.2,52.4,53.5,123.4,123.5,125.8,126.2,126.3,127.4$, $127.7,128.7,128.9,129.5,131.9,134.5,141.2,141.5$.

(R)-3-(1-Phenylethylamino)-propionic acid methyl ester (6p). According to the procedure described in the literature. ${ }^{8}$ Yield $92 \%$. bp $120-130{ }^{\circ} \mathrm{C}, 0.5 \mathrm{mmHg},[\alpha]_{\mathrm{D}}^{20}=+40.5^{\circ}(c=1.2$, $\left.\mathrm{CHCl}_{3}\right)\left[\right.$ Lit. $^{7}[\alpha]_{\mathrm{D}}^{20}=-42.0\left(c=1, \mathrm{CHCl}_{3}\right)$, for $S$ enantiomer $]$.

\section{References:}

1. Hulst, R.; Vries, N. K.; Feringa, B. L. Tetrahedron: Asymmetry 1994, 5, 699-708.

2. Juaristi, E.; Hernández-Rodríguez, M.; López-Ruiz, H.; Aviña, H.; Muñoz-Muñiz, O. Helv. Chim. Acta 2002, 85, 1999-2008. 
3. (a) A. de Parrodi, C.; Moreno, G. E.; Quintero, L.; Juaristi, E. Tetrahedron: Asymmetry 1998, 9, 2093-2099. (b) A. de Parrodi, C.; Juaristi, E.; Quintero, L.; Clara-S, A. Tetrahedron: Asymmetry 1997, 8, 1075-1082.

4. Juaristi, E.; Murer, P.; Seebach, D. Synthesis 1993, 1243-1246.

5. (a) Overberger, C. G.; Marullo, N. P.; Hiskey, R. G. J. Am. Chem. Soc. 1961, 83, 1374-1378. (b) Eleveld, M. B.; Hogeveen, H.; Schudde, E. P. J. Org. Chem. 1986, 51, 3635-3642.

6. Liu, X.; Li, H; Deng, L. Org. Lett. 2005, 7, 167-169.

7. (a) Aldrich Handbook, 2005-2006, 301. (b) Willoughby, C. A.; Buchwald, S. L. J. Am. Chem. Soc. 1994, 116, 8952. (c) Mao, J.; Baker, D. C. Org. Lett, 1999, 1, 841-843.

8. Gutiérrez-García, V. M.; López-Ruiz, H.; Reyes-Rangel G.; Juaristi, E. Tetrahedron 2001, $57,6487-6496$. 


\section{Selected NMR spectrum:}

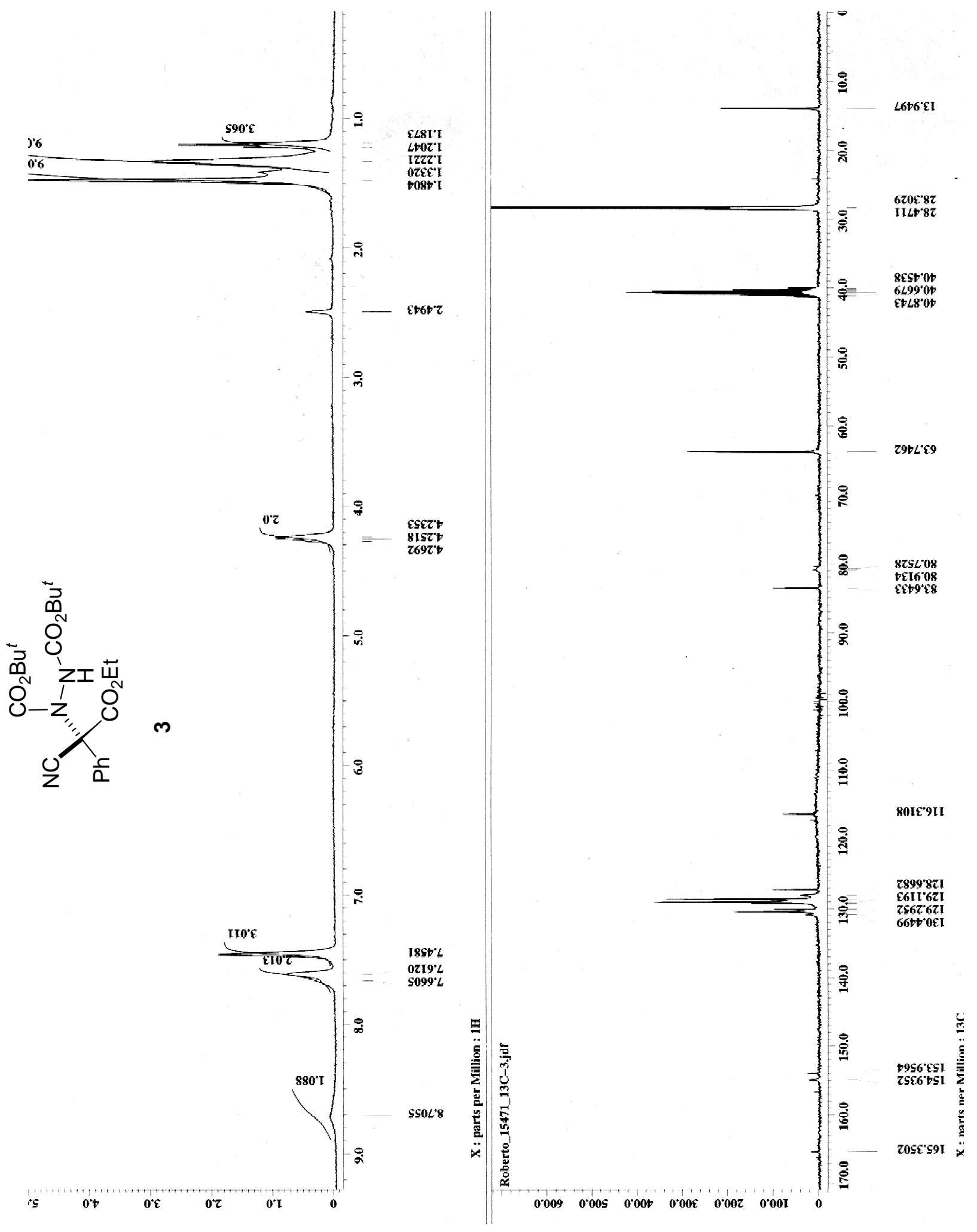




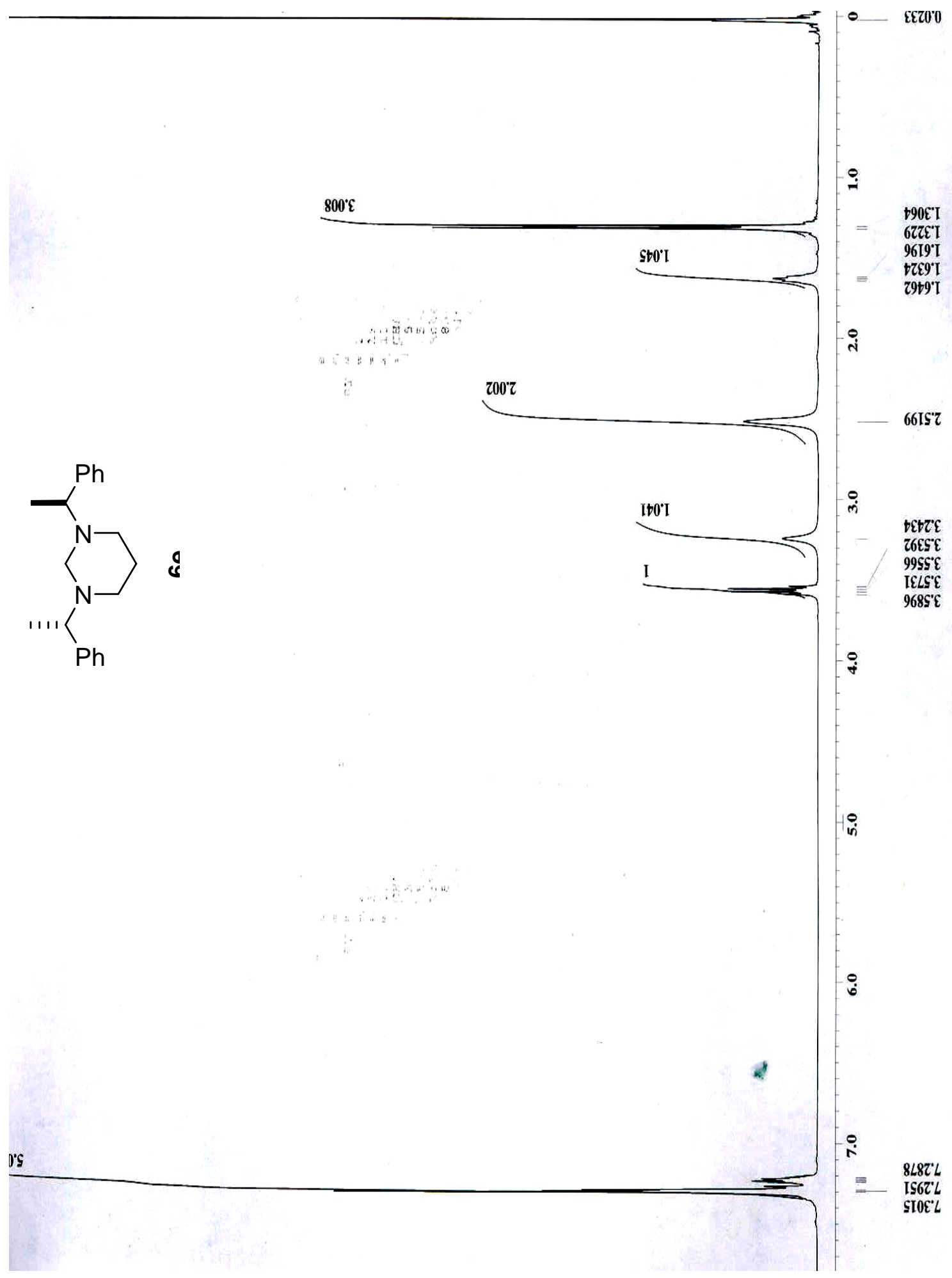



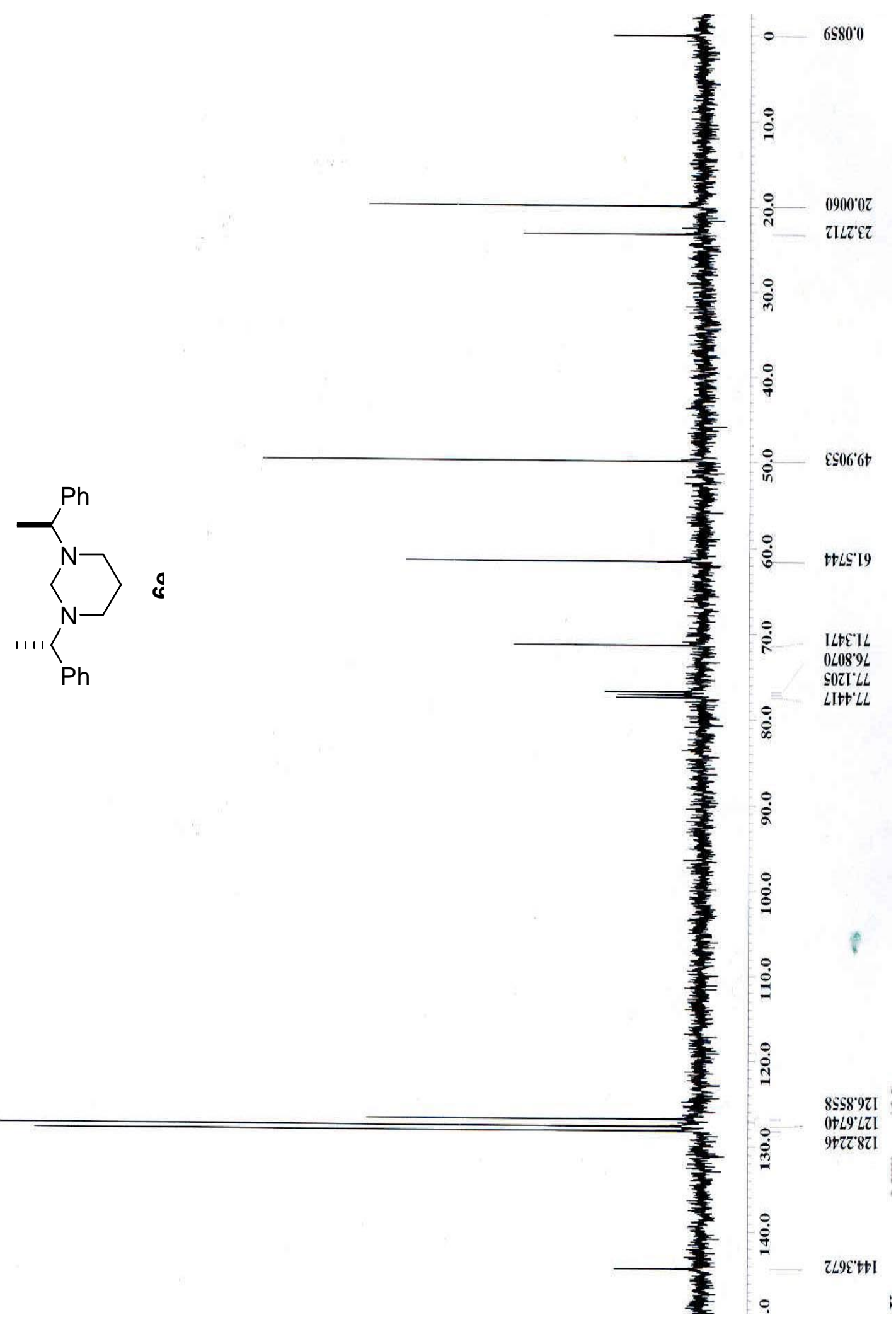


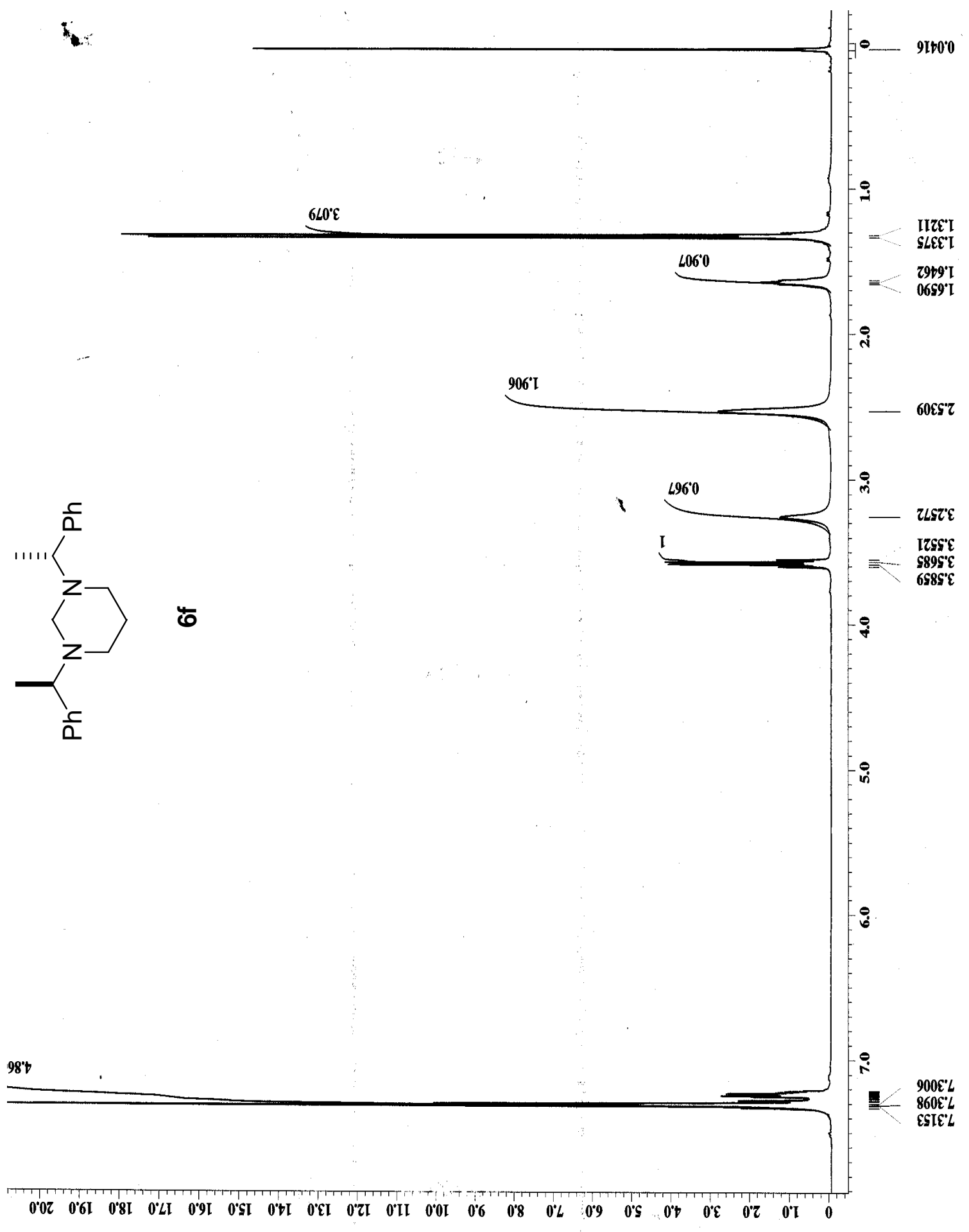




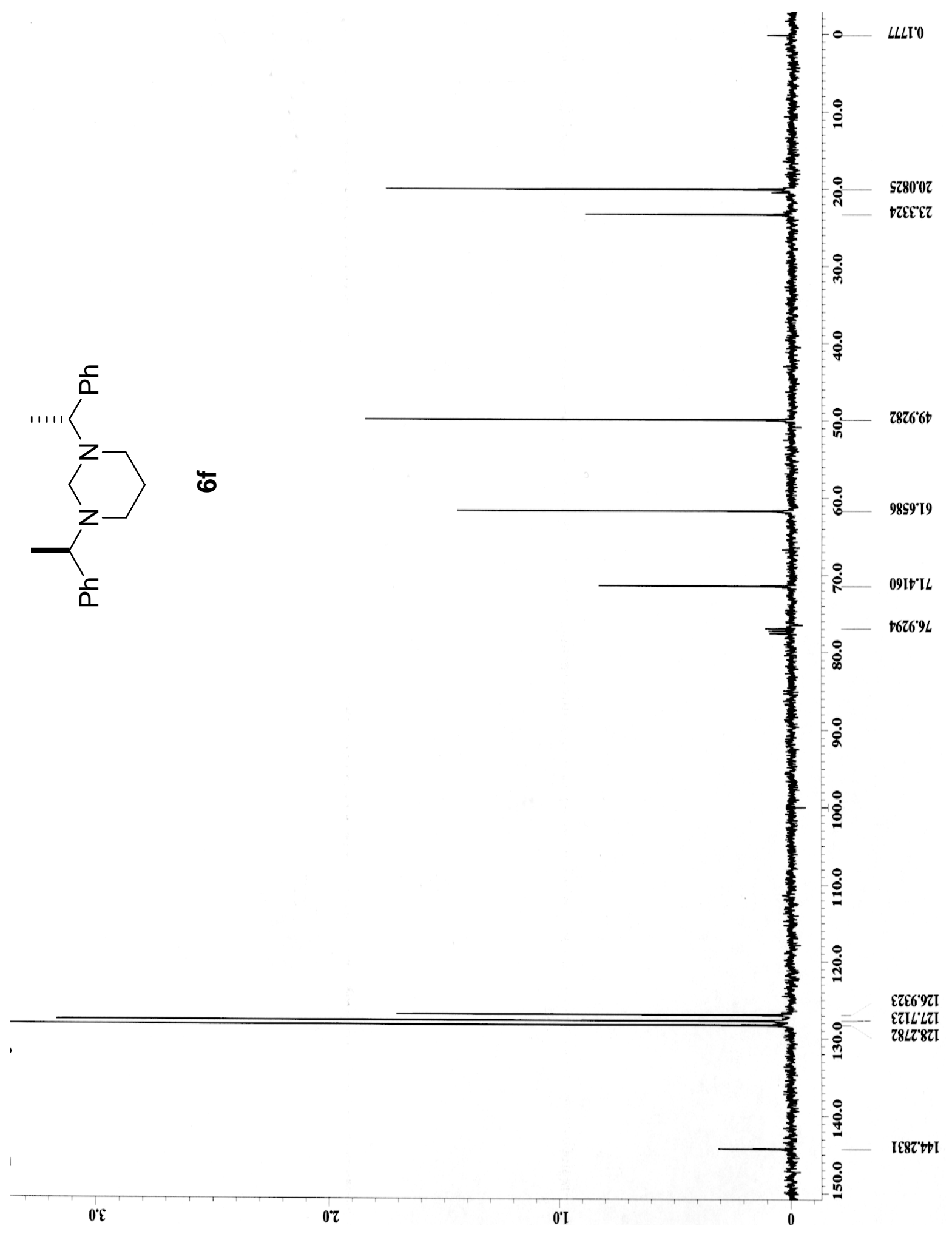




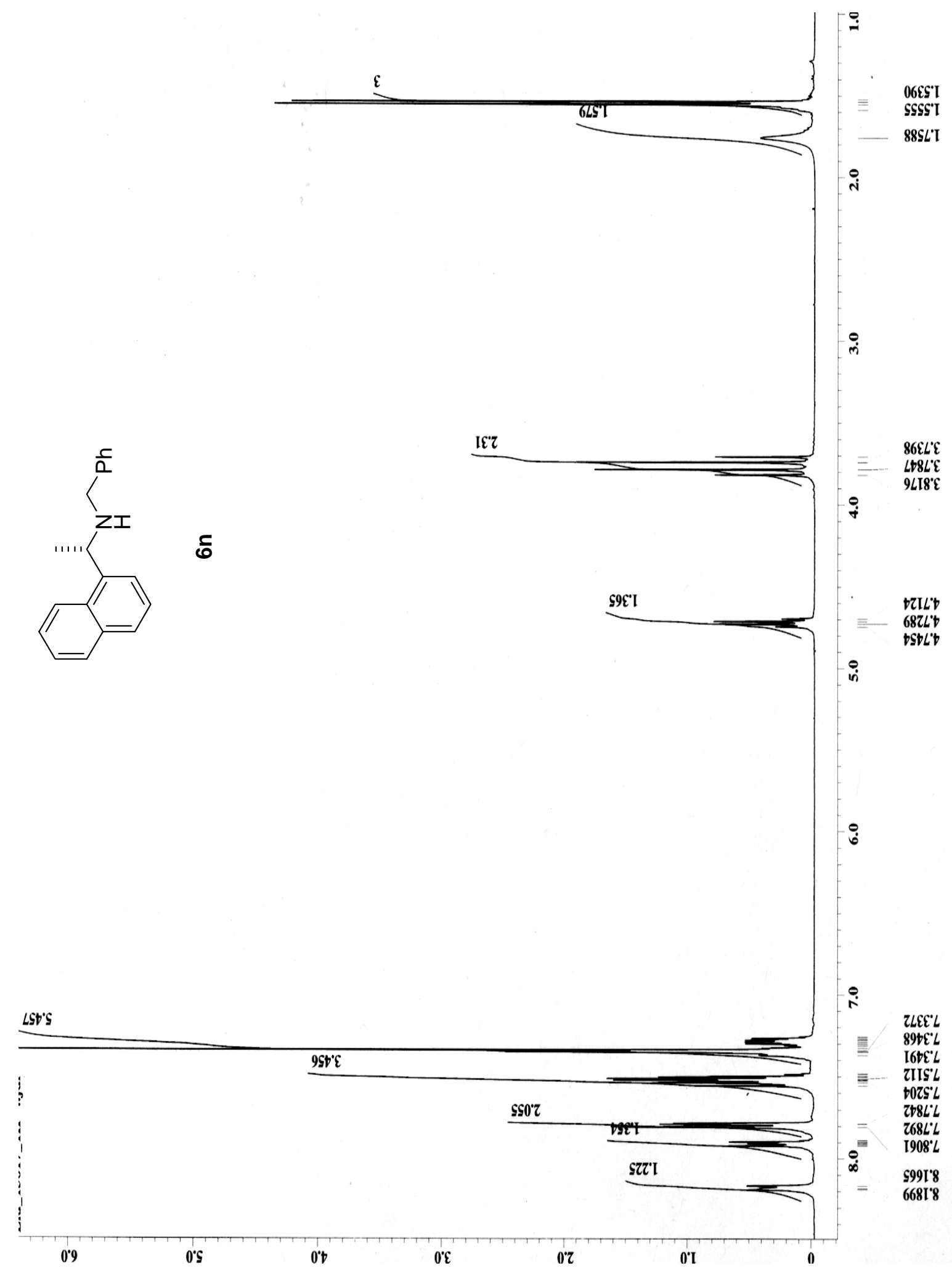


9ES $0 \longrightarrow$
$\angle 99^{\circ} \mathrm{-}$

$00 \partial^{\circ} \nabla 2-$

OEZ 'OE

000. 2S -

EEG $\varepsilon \mathcal{G ~}^{-}$
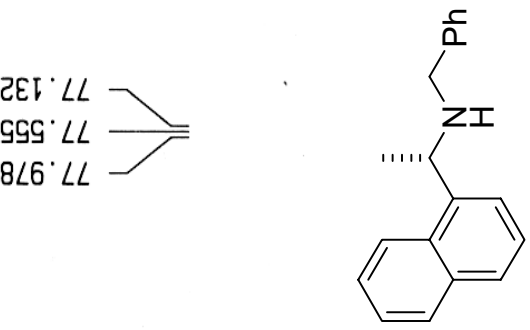

๘
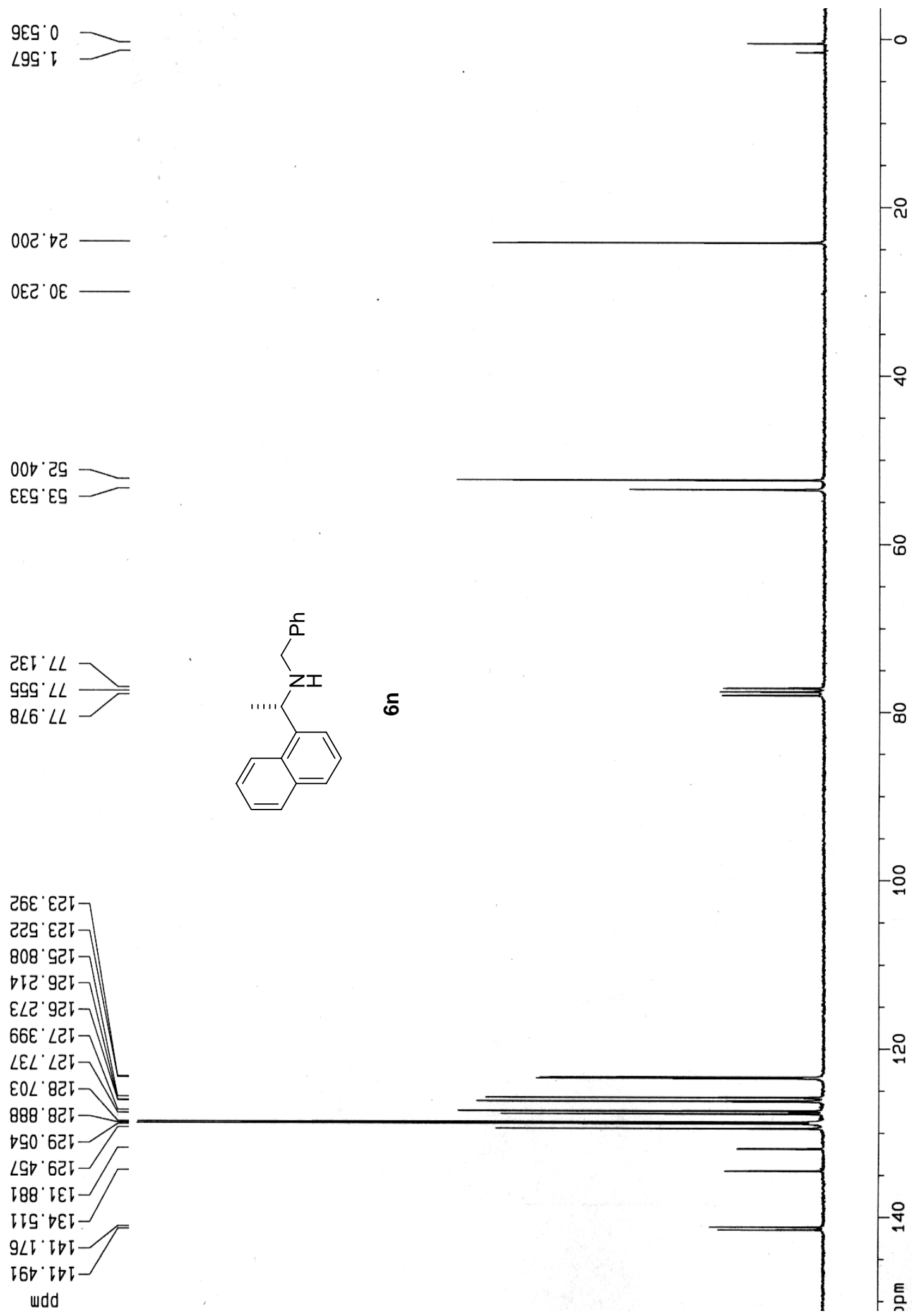
Selected HPLC chromatograms:

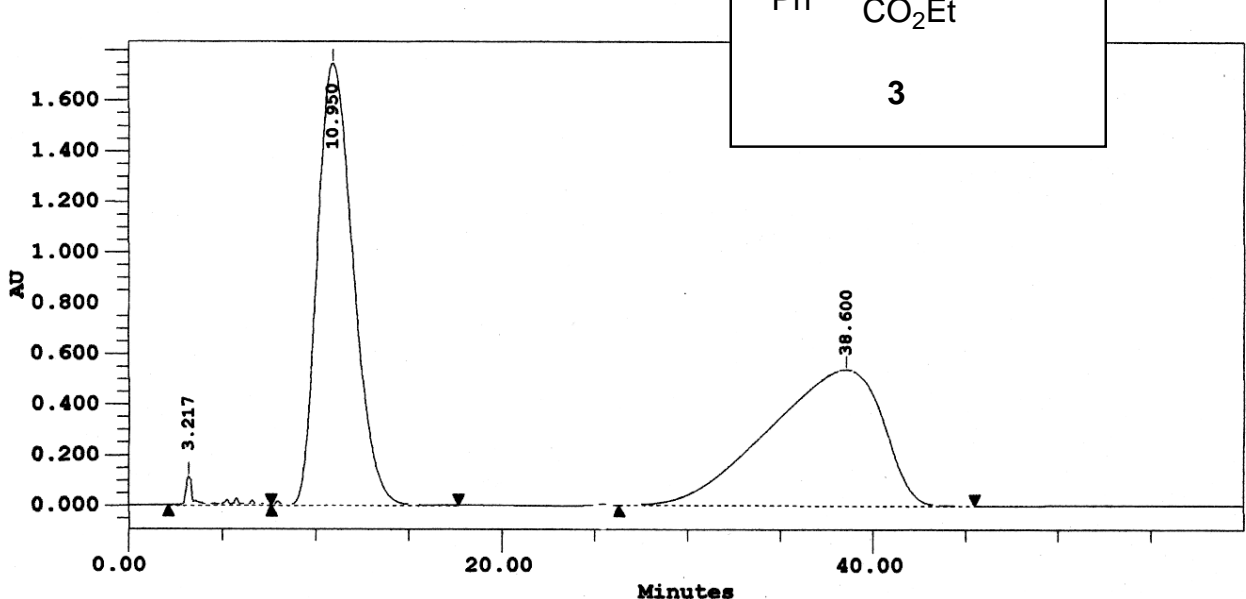

Peak Results

\begin{tabular}{|c|c|c|c|c|c|c|c|c|}
\hline \# & Name & $\begin{array}{l}\text { Ret Time } \\
\text { (min) }\end{array}$ & $\begin{array}{c}\text { Area } \\
\left(\mathrm{uV}^{\star} \text { sec }\right)\end{array}$ & $\begin{array}{l}\text { Height } \\
\text { (uV) }\end{array}$ & $\%$ Area & $8 \mathrm{Ht}$ & $\begin{array}{c}\text { Start Time } \\
\text { (min) }\end{array}$ & $\begin{array}{c}\text { End Time } \\
\text { (min) }\end{array}$ \\
\hline 1 & & 3.217 & 4029777 & 112991 & 0.86 & 4.71 & 2.100 & 7.633 \\
\hline 2 & & 10.950 & 232494914 & 1746314 & 49.89 & 72.83 & 7.633 & 17.700 \\
\hline 3 & & 38.600 & 229493361 & 538407 & 49.25 & 22.46 & 26.333 & 45.533 \\
\hline
\end{tabular}

Table 2, entry 1: with $\mathbf{6 d}$

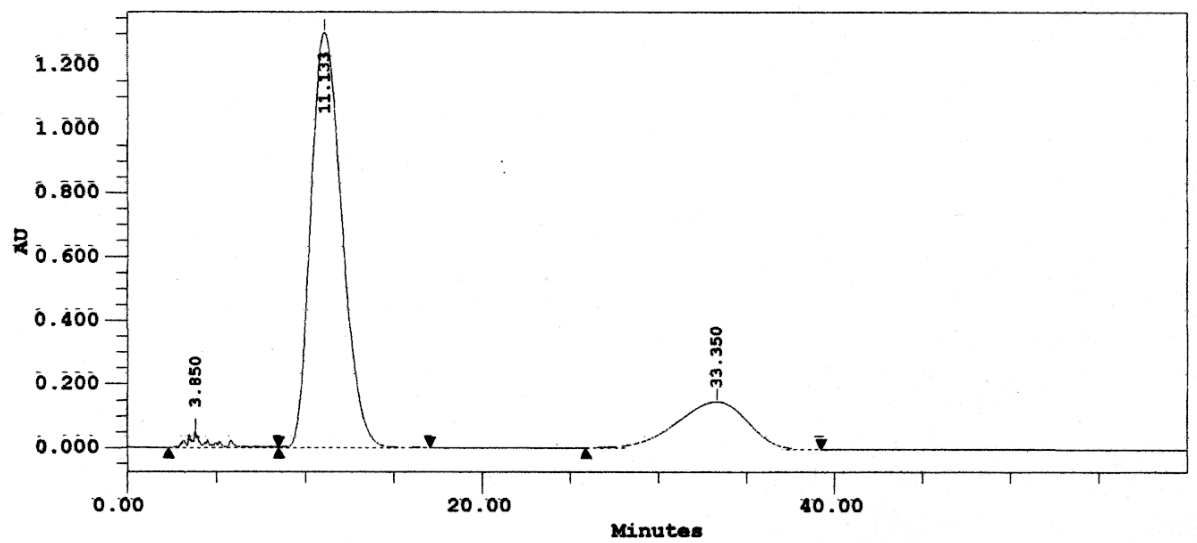

Peak Results

\begin{tabular}{|c|c|c|c|c|c|c|c|c|}
\hline \# & Name & $\begin{array}{c}\text { Ret } \overline{\text { Time }} \\
(\min )\end{array}$ & $\begin{array}{c}\text { Àrea } \\
\left(\mathrm{uV}^{\star} \text { sec }\right)\end{array}$ & $\begin{array}{l}\text { Ḧeight } \\
\text { (uV) }\end{array}$ & \& Area & $8 \mathrm{Ht}$ & $\begin{array}{c}\overline{\text { ștart Time }} \\
(\text { min) }\end{array}$ & $\begin{array}{c}\overline{\text { End Time }} \\
\text { (min) }\end{array}$ \\
\hline 1 & & 3.850 & 3074731 & 50597 & 1.51 & 3.37 & 2.317 & 8.500 \\
\hline 2 & & 11.133 & 158892475 & 1303581 & 78.20 & 86.80 & 8.500 & 17.050 \\
\hline 3 & & 33.350 & 41211399 & 147678 & 20.28 & 9.83 & 25.900 & 39.250 \\
\hline
\end{tabular}

$80 \%$ ee $(-)$ 
Table 2, entry 5: with $6 d$

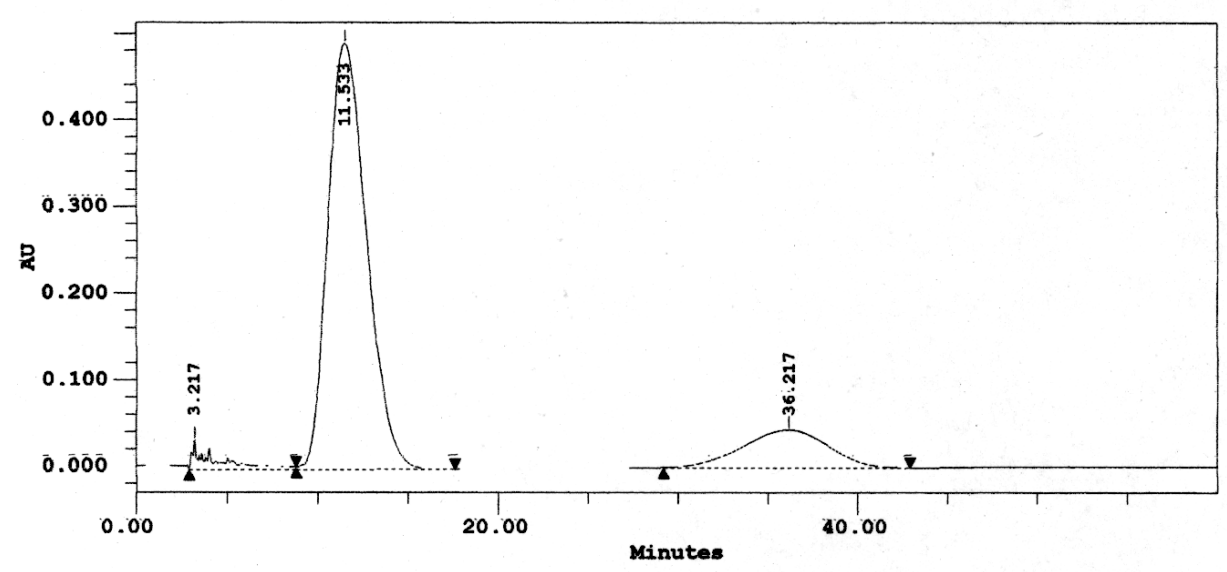

Peak Results

\begin{tabular}{|c|c|c|c|c|c|c|c|c|}
\hline \# & Name & $\begin{array}{c}\text { Ret Time } \\
\text { (min) }\end{array}$ & $\begin{array}{c}\text { Area } \\
\left(u V^{\star} \text { sec }\right)\end{array}$ & $\begin{array}{l}\text { Height } \\
\text { (uV) }\end{array}$ & \& Area & $8 \mathrm{Ht}$ & $\begin{array}{c}\text { Start Time } \\
(\min )\end{array}$ & $\begin{array}{l}\text { End Time } \\
\text { (min) }\end{array}$ \\
\hline 1 & & 3.217 & 2561969 & 35658 & 2.84 & 6.24 & 2.900 & 8.833 \\
\hline 2 & & 11.533 & 73338251 & 491688 & 81.16 & 86.10 & 8.833 & 17.633 \\
\hline 3 & & 36.217 & 14461202 & 43745 & 16.00 & 7.66 & 29.200 & 42.933 \\
\hline
\end{tabular}

\section{$84 \%$ ee $(-)$}

Table 2 entry 7: with $\mathbf{6 m}$

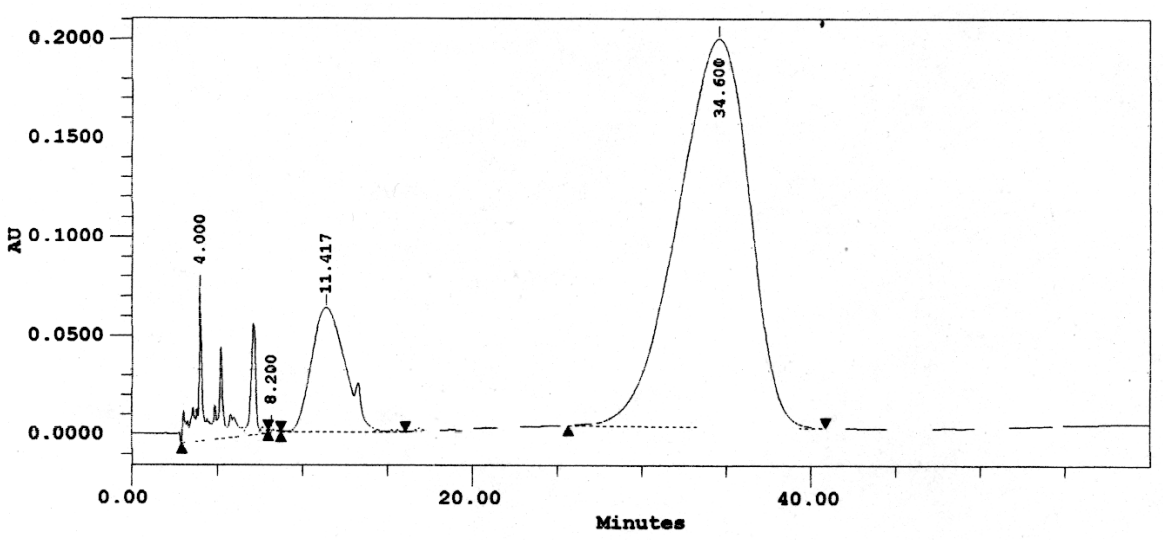

Peak Results

\begin{tabular}{|c|c|c|c|c|c|c|c|c|}
\hline$\#$ & Name & $\begin{array}{l}\text { Ret Time } \\
\text { (min) }\end{array}$ & $\begin{array}{c}\text { Area } \\
\left(\mathrm{uV}^{\star} \mathrm{sec}\right)\end{array}$ & $\begin{array}{l}\text { Height } \\
\text { (uV) }\end{array}$ & \& Area & $8 \mathrm{Ht}$ & $\begin{array}{l}\text { Start Time } \\
(\text { min) }\end{array}$ & $\begin{array}{l}\text { End Time } \\
\text { (min) }\end{array}$ \\
\hline 1 & & 4.000 & 4020361 & 77038 & 5.70 & 22.78 & 2.900 & 7.983 \\
\hline 2 & & 8.200 & 46146 & 2094 & 0.07 & 0.62 & 7.983 & 8.733 \\
\hline 3 & & 11.417 & 8793822 & 62762 & 12.47 & 18.56 & 8.733 & 16.067 \\
\hline 4 & & 34.600 & 57632820 & 196224 & 81.76 & 58.03 & 25.667 & 40.883 \\
\hline
\end{tabular}

$74 \%$ ee (t) 
Table 2, entry 11: with $6 \mathbf{m}$

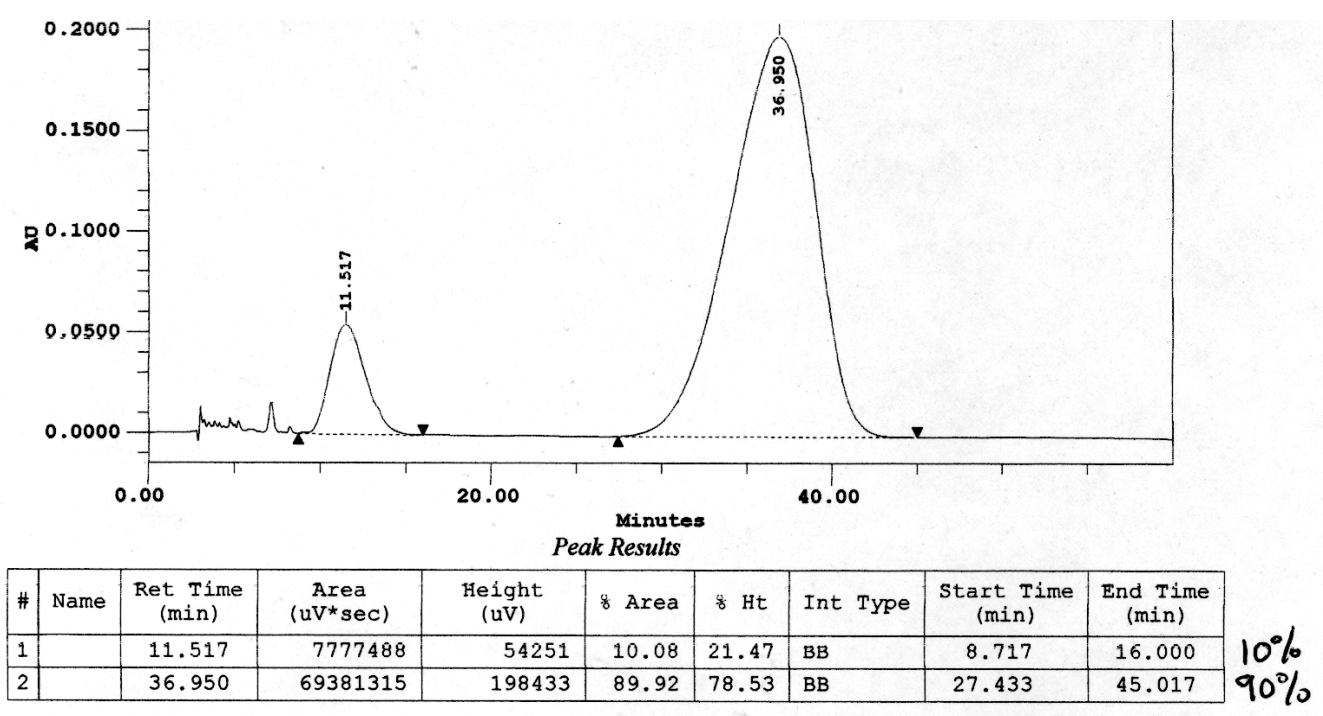

\section{$80 \%$ oce (t)}

Table 3, entry 1: with $6 \mathbf{m}$

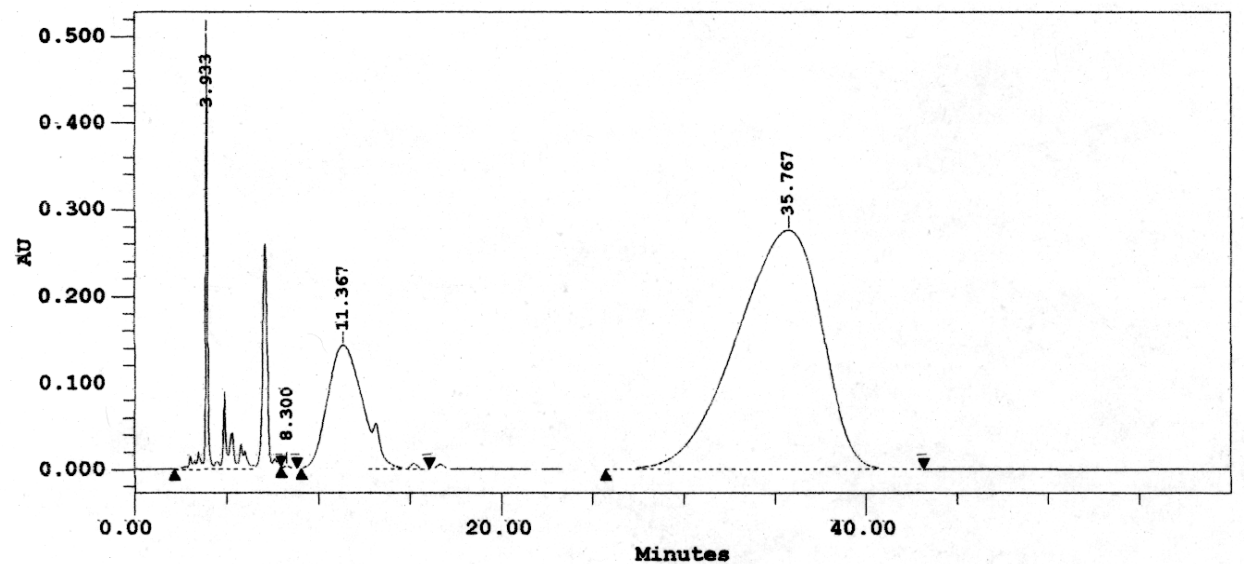

Peak Results

\begin{tabular}{|l|l|r|r|r|r|r|r|r|}
\hline$\#$ & Name & $\begin{array}{c}\text { Ret Time } \\
(\mathrm{min})\end{array}$ & $\begin{array}{c}\text { Area } \\
(\mathrm{uV} \text { (sec) }\end{array}$ & $\begin{array}{c}\text { Height } \\
\text { (uV) }\end{array}$ & \& Area & \& Ht & $\begin{array}{c}\text { Start Time } \\
(\mathrm{min})\end{array}$ & $\begin{array}{c}\text { End Time } \\
(\mathrm{min})\end{array}$ \\
\hline 1 & & 3.933 & 10772326 & 502619 & 8.99 & 54.45 & 2.167 & 7.983 \\
\hline 2 & & 8.300 & 55558 & 2502 & 0.05 & 0.27 & 7.983 & 8.850 \\
3 & & 11.367 & 19285754 & 142349 & 16.10 & 15.42 & 9.083 & 16.067 \\
\hline 4 & & 35.767 & 89655978 & 275671 & 74.86 & 29.86 & 25.717 & 43.183 \\
$18 \%$ \\
$82 \%$
\end{tabular}

$64 \%$ ee $(t)$ 